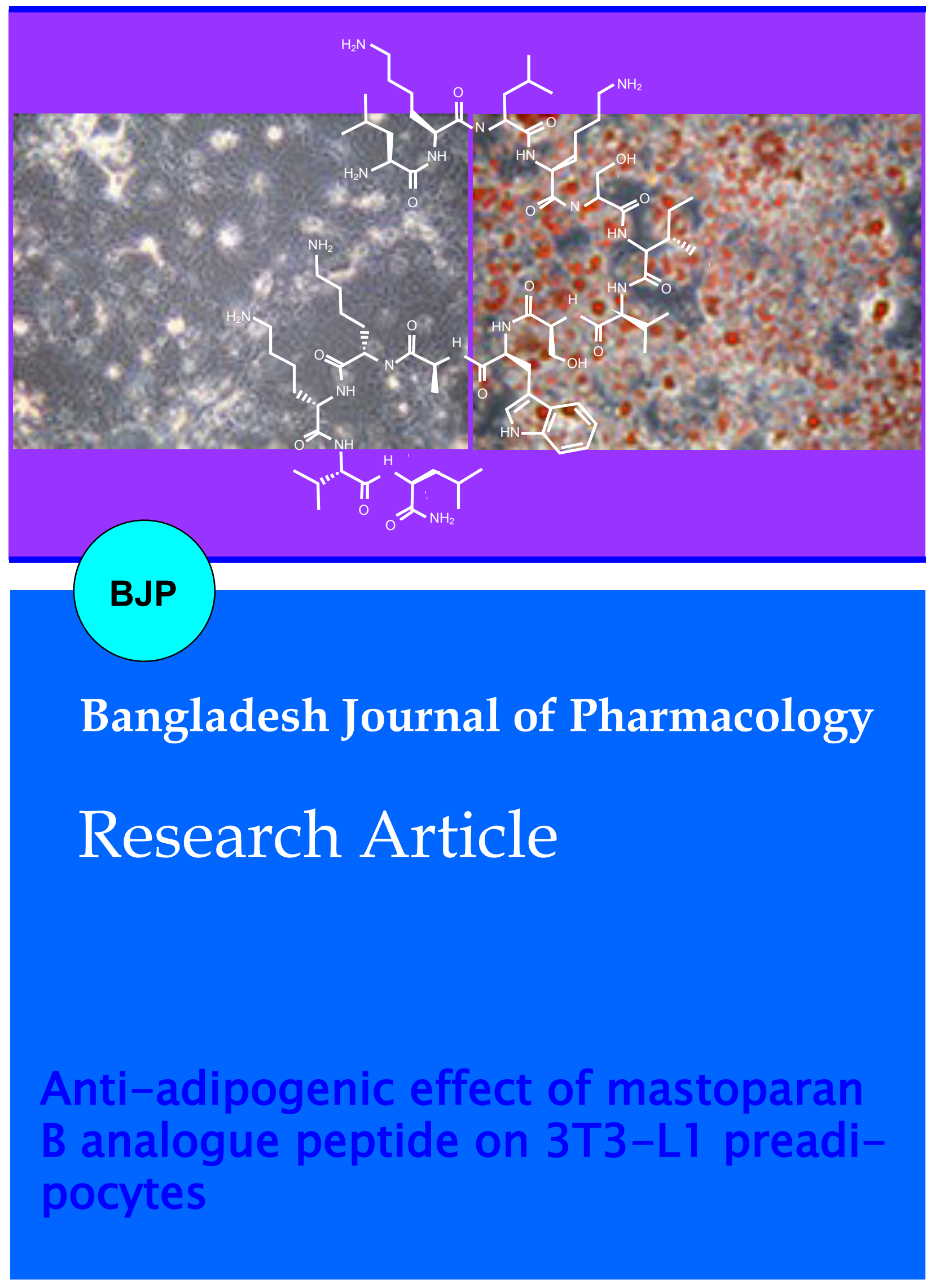




\title{
Anti-adipogenic effect of mastoparan B analogue peptide on 3T3-L1 preadipocytes
}

\author{
Ji Hye Kim¹,2, Mi Jeong Jo', Hye Jin Go3, Nam Gyu Park³ and Gun Do Kim \\ ${ }^{1}$ Department of Microbiology, College of Natural Sciences, Pukyong National University, Busan 48513, Korea; \\ ${ }^{2}$ Department of Biotechnology, Laboratory of Stem Cells and Tissue Regeneration, College of Life Sciences and \\ Biotechnology, Korea University, Seoul 02841, Korea; ${ }^{3}$ Department of Biotechnology, College of Fishery Sciences, \\ Pukyong National University, Busan 48513, Korea.
}

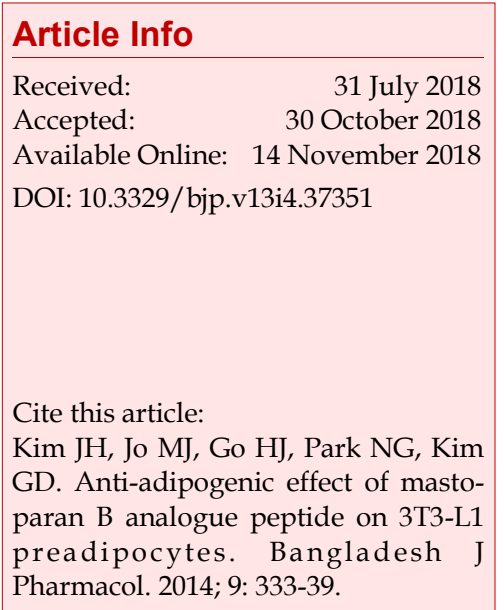

\begin{abstract}
Mastoparan B (MP-B), a cationic tetradecapeptide isolated from the venom of the Vespa basalis, exhibits cardiovascular effects, local edema and antibacterial activity. In this study, the anti-adipogenic effect of an MP-B analogue and its mechanism of action in 3T3-L1 preadipocytes were studied. The MP-B analogue (MP-B12) inhibited preadipocyte differentiation and decreased the expression of adipogenic transcription factors, including CCAAT/enhancer binding protein-alpha $(\mathrm{C} / \mathrm{EBPa})$, nuclear receptor peroxisome proliferatoractivated receptor gamma (PPARY) and sterol regulatory element-binding protein-1 (SREBP-1). Moreover, MP-B12 regulated the phosphorylation of Akt and glycogen synthase kinase- 3 beta (GSK-3 $\beta$ ), both of which play a role in preadipocyte differentiation, in which insulin and certain growth factors stimulated adipogenesis. This study demonstrates that MP-B12 inhibits preadipocyte differentiation and the accumulation of lipid droplets in 3T3-L1 preadipocytes and could potentially be used to treat obesity.
\end{abstract}

\section{Introduction}

Adipose tissue plays a crucial role in the energy storage, lipid homeostasis and fatty acid release. Obesity occurs when the energy intake exceeds the energy expenditure, and this disease is epidemic both in the developed and developing countries (Ogden et al., 2014; Zimmet et al., 2014). Obesity is defined as increased fat mass due to an increase in the number and size of adipocytes. Obesity leads to metabolic complications, including type II diabetes, insulin resistance, hyperlipidemia, hypertension, coronary heart disease and cancer (Spiegelman and Flier, 1996).

Several drugs have been used to treat obesity, such as orlistat, sibutramine, dinitrophenol, and thyroid hormone. These drugs can cause serious adverse effects, including dry mouth, insomnia, anorexia and valvular heart disease (Halford, 2006). Thus, research aimed to find out the healthy food or drug without adverse effects that is required for the prevention and treatment of obesity (Ho et al., 2013). A mouse preadipocyte cell line, 3T3-L1, has been commonly used in an in vitro model system to investigate the molecular mechanism of adipogenesis (Kwak et al., 2013). 3T3-L1 preadipocytes differentiate upon exposure to inducers, such as insulin, 3-isobutyl-1-methylxanthine and dexamethasone (Kanda et al., 2012). This treatment initiates early events in adipogenesis, which depend on the coordinated regulation of gene expression (Ho et al., 2013; Lee et al., 2013). Adipogenic transcription factors, such as C/EBPa, PPARY and SREBP-1, are the key regulators of adipogenesis (Kang et al., 2013a). These factors cooperate to promote the expression of adipogenic genes that cause terminal differentiation of 
preadipocytes (Lee et al., 2013). The Akt signaling pathway is important for the preadipogenic effects of insulin and for promoting adipocyte differentiation through increasing PPARy expression (Kwak et al., 2013). Mastoparan, the mast-cell degranulating peptide of vespid venoms, is the major peptide component of venom in many species of hornets (Ho et al., 2001).

Mastoparan B (MP-B) is a cationic tetradecapeptide (LKLKSIVSWAKKVL-NH $\mathrm{N}_{2}$ ) isolated from the venom of the Vespa basalis hornet of Taiwan (Kang et al., 2013b). The MP-B peptide has a wide-range of biological activities, including stimulation of histamine release, erythrocyte lysis and antimicrobial activity against bacteria (Gwon et al., 2013). However, the anti-obesity effect of MP-B has not been described. We constructed an MP-B analogue peptide (MP-B12, LKLKSIVSWAKAVS- $\mathrm{NH}_{2}$ ), which is a derivative of MP -B with an Ala substitution at the $12^{\text {th }}$ residue. In this study, we evaluated the inhibitory effects of MP-B and MP-B12 on adipogenesis, and investigated how MP-B12 acts to reduce the differentiation of preadipocytes into adipocytes.

\section{Materials and Methods}

\section{Peptide synthesis}

Following identification by sequencing, nine peptides were synthesized using the solid-phase synthesis method on an ASP48S pepsynthesizer using 9-fluorenylmethoxycarbonyl (Fmoc)-polypeptide active ester chemistry in Peptron Inc, Korea (Table I). Each analogue's residue was replaced by Ala.

\section{Table I}

Primary structure of MP-B and its analogues

\begin{tabular}{|lc|}
\hline Peptide & \multicolumn{2}{c|}{ Sequence } \\
\hline $\begin{array}{l}\text { Mastoparan B } \\
\text { (MP-B) }\end{array}$ & L-K-L-K-S-I-V-S-W-A-K-K-V-L-NH ${ }_{2}$ \\
MP-B1 & A-K-L-K-S-I-V-S-W-A-K-K-V-L-NH ${ }_{2}$ \\
MP-B3 & L-K-A-K-S-I-V-S-W-A-K-K-V-L-NH ${ }_{2}$ \\
MP-B6 & L-K-L-K-S-A-V-S-W-A-K-K-V-L-NH ${ }_{2}$ \\
MP-B7 & L-K-L-K-S-I-A-S-W-A-K-K-V-L-NH ${ }_{2}$ \\
MP-B11 & L-K-L-K-S-I-V-S-W-A-A-K-V-L-NH ${ }_{2}$ \\
MP-B12 & L-K-L-K-S-I-V-S-W-A-K-A-V-L-NH ${ }_{2}$ \\
MP-B13 & L-K-L-K-S-I-V-S-W-A-K-K-A-L-NH ${ }_{2}$ \\
MP-B14 & L-K-L-K-S-I-V-S-W-A-K-K-V-A-NH ${ }_{2}$ \\
\hline
\end{tabular}

\section{Cell culture and differentiation}

3T3-L1 preadipocytes were purchased from the American Type Culture Collection (USA). The cells were cultured in Dulbecco's modified eagle's medium (Cellgro, Mediatech, Inc., USA) containing 10\% fetal calf serum $\left(\mathrm{Gibco}^{\circledR}, \mathrm{USA}\right)$ and 1\% penicillin/streptomycin
(Cellgro), and incubated at $37^{\circ} \mathrm{C}$ in a humidified atmosphere and $5 \% \mathrm{CO}_{2}$.

To induce differentiation, 3T3-L1 preadipocytes were cultured as previously described (Choi et al., 2015).

\section{Cell cytotoxicity}

3T3-L1 preadipocytes were cultured in 96-well plate and treated with MP-B and MP-B12 for 24 hours. After incubation, $10 \mu \mathrm{L}$ of WST- $1{ }^{\circledR}$ (Daeil Lab Service, Korea) was added to each well, and the plate was incubated at $37^{\circ} \mathrm{C}$ for 3 hours. The absorbance was measured at 460 $\mathrm{nm}$ with an ELISA reader (Molecular Devices, USA).

\section{Oil Red $O$ staining and cell quantification}

On day 9 after the induction of differentiation, the cells were stained with Oil Red O. The cells were washed and fixed in $10 \%$ formalin (Junsei Chemical Co. Ltd, Japan) for 1 hour. The cells were then stained with filtered Oil Red O solution (60\% isopropanol and 40\% water) (Sigma-Aldrich, Japan) for $30 \mathrm{~min}$. After staining, the stained lipid droplets were viewed on an inverted microscope (magnification $\times 200$ ). The stained lipid droplets were dissolved in $100 \%$ isopropanol (to extract intracellular Oil Red O stain) and the absorbance was measured at $520 \mathrm{~nm}$.

\section{Measurement of triglyceride contents}

The intracellular triglyceride contents were measured in 12-well plates using a commercially available triglyceride quantification kit (BioVision, Inc., USA) according to the manufacturer's instructions. On day 9, the treatment medium was removed, and cells were rinsed with PBS. Another $200 \mu \mathrm{L}$ PBS was added to each well, and the contents were homogenized by sonication for 1 min. Total triglycerides were measured using the assay kit.

\section{Western blot analysis}

3T3-L1 preadipocytes were treated with MP-B and MPB12 during adipocyte differentiation. After treatment, cells were collected and lysed in PRO-PREPTM protein extraction solution (iNtRON Biotechnology, Korea). The protein samples were resolved by $12 \%$ SDSpolyacrylamide gel electrophoresis (SDS-PAGE) and transferred to a nitrocellulose membrane (PALL Life Sciences, USA). The membranes were blocked with PBST buffer (0.5\% Tween-20) containing 5\% skim milk. After blocking, the membranes were probed with primary antibodies (Cell Signaling Technology Inc., USA) and then incubated with horseradish peroxidaseconjugated anti-rabbit IgG or anti-mouse IgG as second antibodies. The blots were visualized by an enhanced chemiluminescent (ECL) detection solution (Abfrontier Ltd., Korea).

\section{Statistical analysis}

All statistical analyses were performed using the Prism 
7.0 software (GraphPad, USA) for Windows. Statistical significance of differences between values was calculated for various experimental and control groups. The results are presented as the means \pm SD error of the mean (S.E.M.). An ANOVA post hoc test and subsequent Dunnett's multiple tests were used for additional statistical analyses.

\section{Results}

Comparative effects on adipogenesis of 3T3-L1 preadipocytes

Microscopic analysis of Oil Red O stained 3T3-L1 cells, cultured in the absence and presence of MP-Bs, revealed the accumulation of intracellular lipid droplets. The quantification of the extracted Oil Red O stain showed that the lipid content was strongly reduced by $10 \mu \mathrm{M}$ MP-B12 compared to positive control (Figure 1). Thus, both MP-B (native form) and MP-B12 were used in subsequent experiments in this study.

\section{Differentiation of 3T3-L1 preadipocytes}

MP-B and B12 did not significantly affect the cell viability at concentration of $10 \mu \mathrm{M}$ for 24 hours (Figure 2). To investigate the effect of MP-Bs on adipogenesis, 3T3-L1 cells were induced to differentiate with MDI in the presence or absence of MP-Bs for 9 days. After differentiation, the effect of MP-B on the lipid accumu- lation of adipocytes was measured by Oil Red $\mathrm{O}$ staining (Figure 3A and B). Both MP-B and B12 inhibited the differentiation of 3T3-L1 cells. MP-B12 inhibited lipid accumulation in adipocytes better than

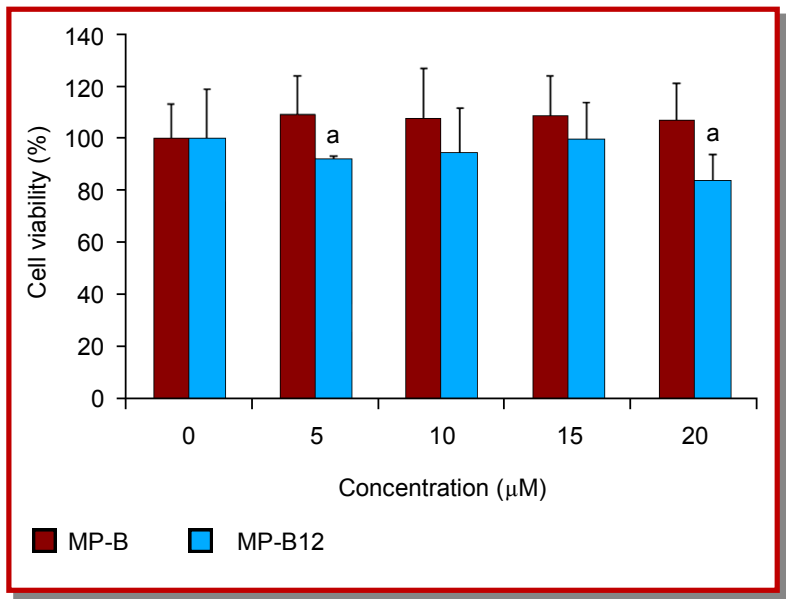

Figure 2: Effect of MP-B peptides on cell viability in 3T3-L1 cells. The cells were cultured with MP-B and MP-B12 (0 20 $\mu \mathrm{M})$. The data were expressed as the percentage of cell viability and represented as the means \pm SE of three experiments in which each treatment was performed in triplicate. ${ }^{a} p<0.05$

MP-B. Furthermore, MP-B12 inhibited the differentiation of 3T3-L1 preadipocytes in a dose-dependent manner, with an $\mathrm{IC}_{50}$ value of $12 \mu \mathrm{M}$. Treatment with

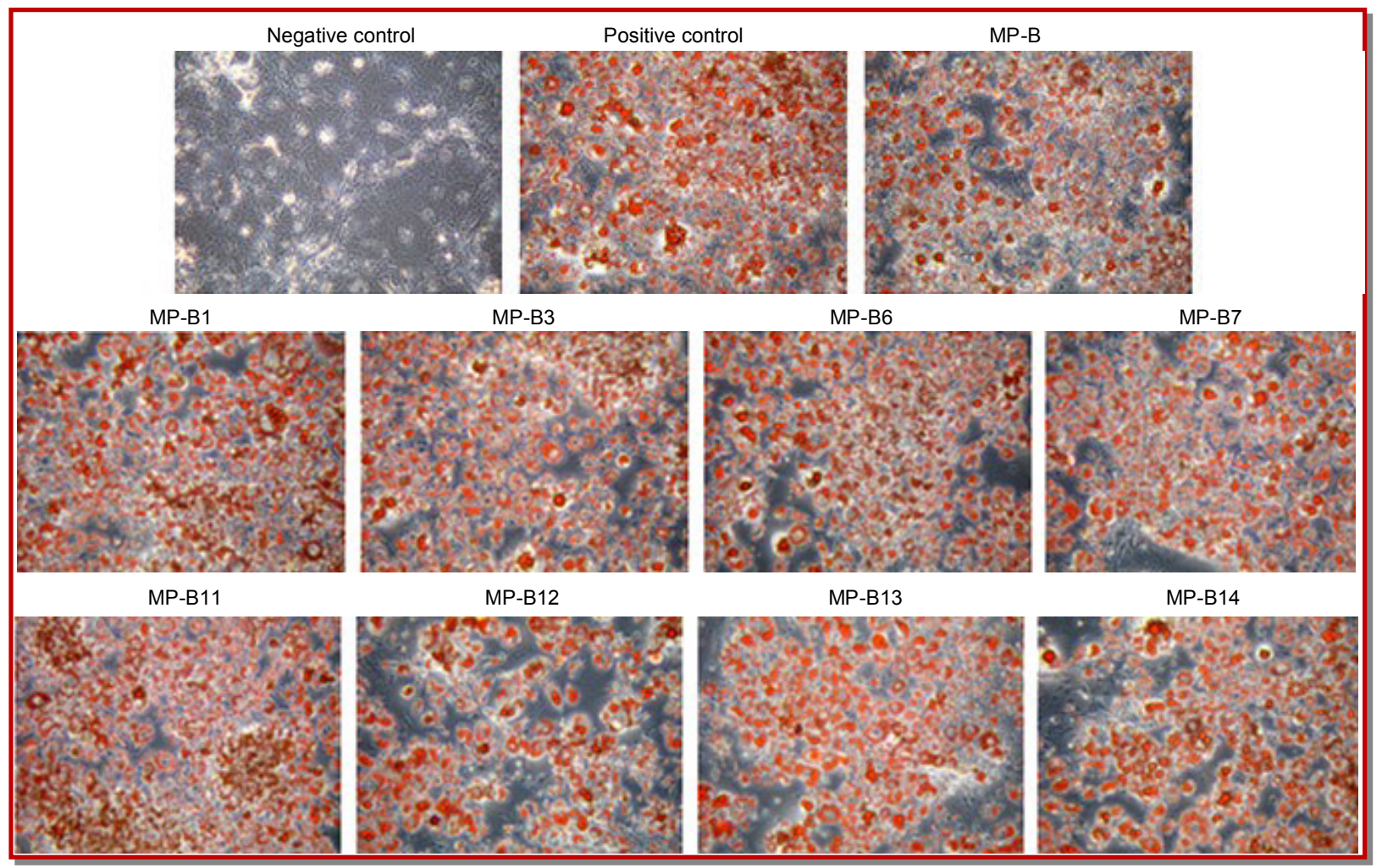

Figure 1: Effect of MP-B and its analogue on intracellular lipid accumulation in 3T3-L1 cells. The cells were cultured with MP-B and its analogue $(10 \mu \mathrm{M})$ and stained by Oil Red $\mathrm{O}$ 
MP-Bs significantly decreased the lipid droplets compared with MDI only-treated cells. These results demonstrate that MP-Bs inhibited the differentiation of preadipocytes. To measure the level of intracellular triglycerides, 3T3-L1 preadipocytes were differentiated with MP-Bs prior to analysis. Figure $3 \mathrm{C}$ shows that MP$\mathrm{B}$ and B12 treatment reduced the triglyceride content and inhibited intracellular triglyceride accumulation in 3T3-L1 cells. Both MP-B and B12 reduced triglyceride content, but the reduction of lipid accumulation by MPB12 was greater than that of MP-B. The inhibitory effects of MP-B12 in triglyceride accumulation during adipogenesis were also in dose-dependent manner.

\section{Expression of adipogenic genes}

As shown in Figure 4, MP-B12 suppressed the expression of SREBP-1 compared with control and MP-B10 treated cells, and indicated that MP-B12 modulated adipogenesis by regulating SREBP-1 protein. SREBP-1 increased the synthesis of triglyceride and affected lipo- genic gene expression, like PPAR $\gamma$ and CEB/Pa in early stage of adipogenesis. We identified that MB-B12 inhibited the expression of PPARY and CEB/Pa proteins. Thus, MP-Bs effectively decreased the expression of key adipogenic protein and confirmed its anti-adipogenic activity.

\section{Regulation of PI3K/Akt pathway}

We investigated the effect of MP-Bs on the PI3K/Akt pathway and found that MP-Bs reduced the expression of PI3K p110 isoforms ( $\alpha, \beta$ and $\gamma$ ) in 3T3-L1 cells (Figure 4). MP-B12 dramatically decreased the expression of PI3K p110 isoforms compared to MP-B10. Also, the phospho-Akt expression was decreased in presence of MP-B12. To investigate the effect of MP-Bs on regulation of Akt, the levels of down-stream mole-cules of Akt signaling were examined. First, we investigated phosphorylation level of ERK44/42 in early stage of adipogenesis, and found its down-regulation as control. Also, a phosphorylation of AMPKa, a regulator of

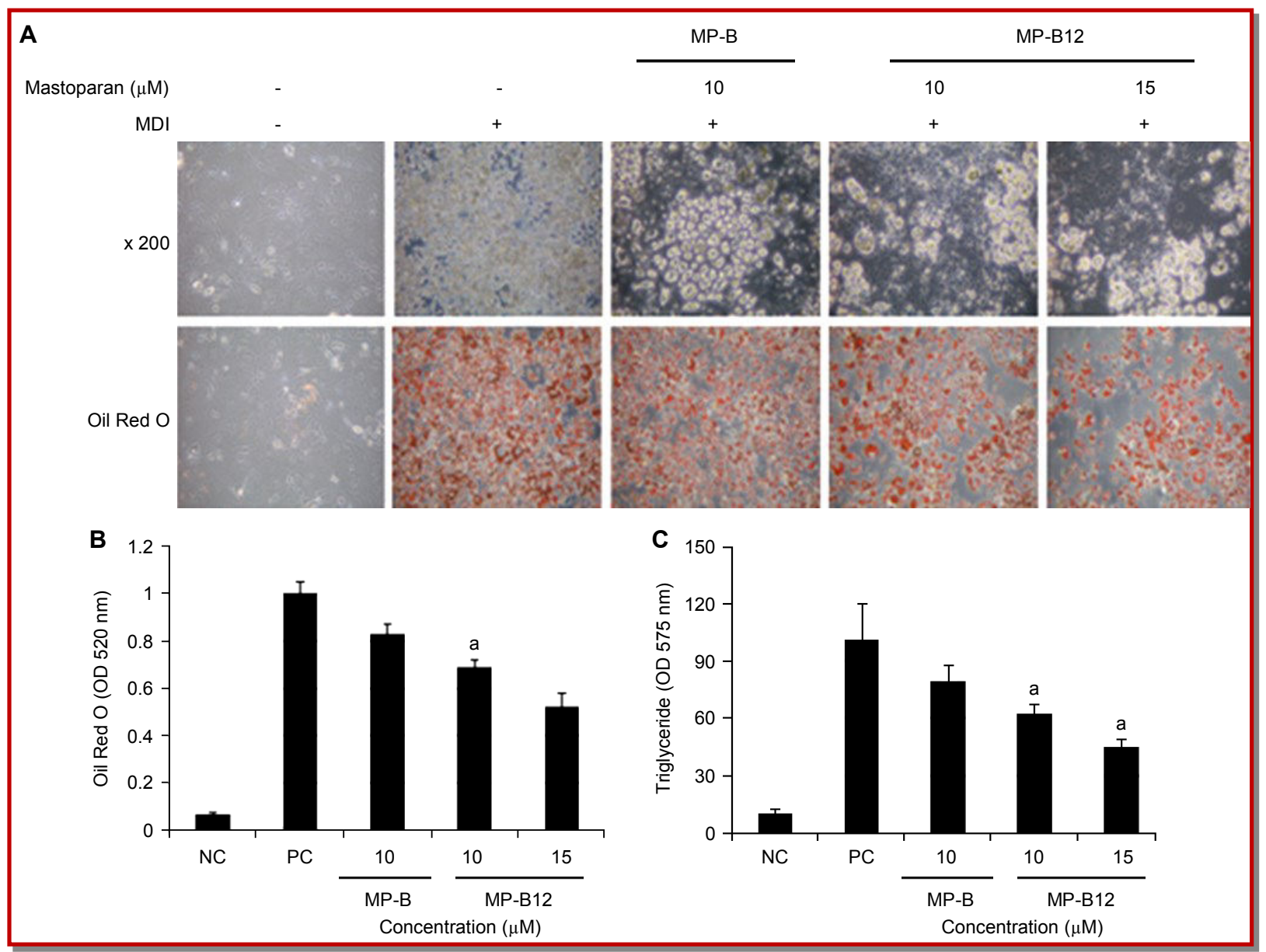

Figure 3: Effect of MP-B peptides on adipogenesis in 3T3-L1 cells. (A) Intracellular lipid accumulation was assessed by Oil Red O staining and observed using an inverted microscope. Oil Red $\mathrm{O}$ stained adipocytes were captured at x200 magnification. (B) The absorbance of Oil Red O dissolved in isopropanol was determined at $520 \mathrm{~nm}$. (C) Lipid accumulation was assessed by measuring intracellular triglyceride levels. Con, 3T3-L1 preadipocytes; MDI, fully differentiated adipocytes; MP-B $10 \mu \mathrm{M}$, fully differentiated adipocytes (MDI + $10 \mu \mathrm{M}$ MP-B); MP-B12 $10 \mu \mathrm{M}$, fully differentiated adipocytes (MDI + $10 \mu \mathrm{M}$ MP-B12); MP-B12 $15 \mu \mathrm{M}$, fully differentiated adipocytes (MDI + $15 \mu \mathrm{M}$ MP-B12) 


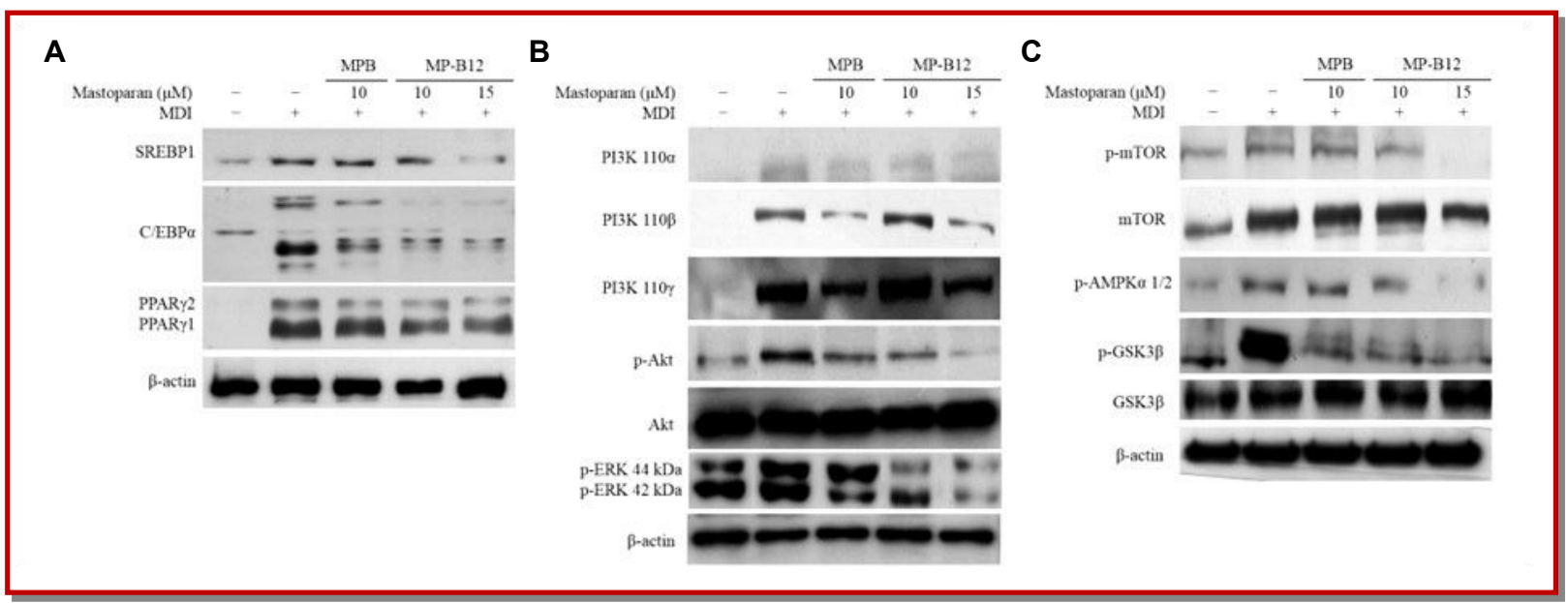

Figure 4: Effect of MP-B peptides on protein expression in 3T3-L1 cells. Differentiation of 3T3-L1 preadipocyte was induced by MDI medium in the absence or presence of MP-B peptides. The expression levels of various proteins were determined by Western blot analysis

lipogenesis, was inhibited by MP-Bs. We then examined the expression of mTOR, an adipogenic gene regulator, and the expression of phospho-mTOR was reduced. Moreover, we analyzed the effects of MP-Bs on GSK3 $\beta$, related with synthesis of fatty acid, and phosphoGSK3 $\beta$ was decreased compared to control.

\section{Discussion}

Over the past several years, the anti-obesity effects of many natural substances have been reported to be mediated through regulation of adipogenesis. However, there are few studies on mastoparan, an antiaidpogenic candidate compound derived from the bee, in the early stage of 3T3-L1 preadipocyte differentiation. In this study, we investigated the overall antiadipogenic effect of mastopran via regulation of SREBP1, PPARY and CEB/Pa gene expression, as well as via the PI3K/Akt pathways.

Our date showed that MP-Bs treatment reduced the amounts of Oli Red O staining in a dose-dependent manner, and microscopic inspection showed a decrease in the level of accumulated intracellular lipid droplets. The triglyceride levels of 3T3-L1 were markedly reduced by treating with MP-Bs. Triglycerides were used as a marker of adipogenesis, as they play an important role in transporting fatty acids and serve as an energy source (Lampe et al., 1983).

Differentiation of preadipocytes into adipocytes is tightly regulated through sequential activation of several transcriptional factors, including $\mathrm{C} / \mathrm{EBPa}, \mathrm{PPAR} \gamma$ and SREBP-1 (Lampe et al., 1983; Song et al., 2013). PPARY and $\mathrm{C} / \mathrm{EBPa}$ are important for the entire terminal differentiation process. Moreover, PPARY is activated by fatty acids, and fat accumulation is associated with PPARY activation. PPARY and C/EBPa activate the expression of genes involved in adipogenesis, such as
aP2, FAS and LPL, to trigger the synthesis of fatty acids and triglycerides (Song et al., 2013). SREBP transcription factors regulate genes related to the metabolism of lipids and cholesterol (Lee et al., 2013). SREBP-1 regulates lipogenic gene expression associated with fatty acid synthesis, which leads to increased synthesis of TG and can contribute to the expression of PPARY ligands (Kang et al., 2013a). These protein expression levels were reduced by MP-Bs. Particularly, PPARY and $\mathrm{C} / \mathrm{EBPa}$ are key adipogenic transcription factors; thus, the protein expression of PPAR $\gamma$ and $C / E B P a$ were decreased more than MP-B, and MP-B12 inhibited protein expression in a dose-dependent manner (Xing et al., 2010). These results demonstrate that MP-Bs suppress adipogenesis through the down-regulation of adipogenic transcription factors, and the inhibitory effect of MP-B12 is greater than that of MP-B.

The activation of extracellular signal regulated kinase $1 / 2(E R K 1 / 2)$ is necessary for adipogenesis (Gwon et al., 2013). The ERK1/2 pathway is upstream of PPARY and C/EBPa. Activation of PPARY and C/EBPa is promoted by ERK activation during 3T3-L1 preadipocyte differentiation (Kwak et al., 2014). ERK1/2 regulates the expression of AMP-activated protein kinase alpha (AMPKa). AMPKa functions as a sensor of cellular energy status in skeletal muscle and is involved in glycogen breakdown, glycolysis, glucose uptake and fatty acid oxidation, in response to changes in gene expression (Zhang et al., 2013). In the signaling pathway, AMPKa is upstream of peroxisome proliferatoractivated receptor $Y$ (PPARY) and inhibits the differentiation of preadipocytes into adipocytes. In this study, MP-B peptides markedly decreased the phosphorylation of ERK1/2 and AMPKa. Moreover, MP-B12 inhibits the expression of ERK1/2 and AMPK- $\alpha$ phosphorylation in a dose-dependent manner and greater than that of MP-B. Because the phosphorylation of AMPK is increased during the early phase of differen- 
tiation, our data suggest that MP-B peptides inhibit adipogenesis via AMPK signaling in the early phase of differentiation.

One of the established signaling mechanisms for increasing PPARY gene expression is mediated by Akt in adipocyte differentiation. Akt plays a crucial role for glucose regulation and lipid metabolism in insulin signaling, and stimulates GSK3 $\beta$, a down-stream target of Akt. In this study, the effects of MP-Bs on phosphorylated Akt were assessed during adipocyte differentiation of 3T3-L1 cells. Treatment with MP-Bs suppessed the MDI-induced phosphorylation of Akt and its upstream signals, including PI3K p110 isoforms $(a, \beta, \gamma)$ and mTOR. The PI3K signaling pathway plays an important role in regulating adipose mass, obesity, and diabetes. PI3K is involved in a signal transduction system down-stream of the insulin receptor (IR) (Zhang et al., 2013). Glycogen synthesis kinase 3 beta (GSK3 $\beta$ ) is a critical down-stream signaling protein of the phosphoinositide 3-kinase (PI3K)/Akt pathway (Song et al., 2013). Insulin signaling activates Akt through PI3K and induces serine/threonine phosphorylation of down-stream target GSK3 $\beta$ (Song et al., 2013). Phosphorylation of insulin-stimulated Akt was reduced after treatment with MP-B and B12 decreased the level of phosphor-Akt in a dose-dependent manner. In addition, insulin-stimulated phosphorylation of GSK3 $\beta$ decreased with the addition of MP-Bs. These results demonstrate that MP-Bs inhibit the phosphorylation of Akt, which suppresses phosphorylation of its substrate kinase GSK3 $\beta$. In the case of PI3K p110 isoforms, MP-B suppressed expression greater than MP-B12. MP-B specifically inhibits expression of PI3K and MP-B12 reduced cellular lipid accumulation in a dosedependent manner in 3T3-L1 cells.

\section{Conclusion}

Treatment with MP-Bs attenuated lipid differentiation in cells, as determined by Oil Red $\mathrm{O}$ staining and a triglyceride accumulation assay, without causing cytotoxicity. Furthermore, MP-B12 has a greater antiadipogenic effect than MP-B, which is achieved through the down-regulation of C/EBPa, PPAR $\gamma$, and SREBP-1 expression and phosphorylation of ERK1/2, AMPKa and the Akt signaling pathway. These data collectively indicate that MP-Bs, especially MP-B12, are potent inhibitors of adipocyte differentiation.

\section{Conflict of Interest}

The author declare no conflict of interest.

\section{References}

Choi JS, Kim JH, Ali MY, Jung HJ, Min BS, Choi RJ, Kim GD,
Jung HA. Anti-adipogenic effect of epiberberine is mediated by regulation of the Raf/MEK $1 / 2 /$ ERK $1 / 2$ and AMPKalpha/Akt pathways. Arch Pharm Res. 2015; 38: 215362.

Gwon SY, Ahn JY, Jung CH, Moon BK, Ha TY. Shikonin suppresses ERK 1/2 phosphorylation during the early stages of adipocyte differentiation in 3T3-L1 cells. BMC Complement Altern Med. 2013; 13: 1-8.

Halford JC. Pharmacotherapy for obesity. Appetite 2006; 46: 610.

Ho CL, Shih YP, Wang KT, Yu HM. Enhancing the hypotensive effect and diminishing the cytolytic activity of hornet mastoparan B by D-amino acid substitution. Toxicon 2001; 39: 1561-66.

Ho JN, Son ME, Lim WC, Lim ST, Cho HY. Germinated brown rice extract inhibits adipogenesis through the downregulation of adipogenic genes in 3T3-L1 adipocytes. Plant Foods Hum Nutr. 2013; 68: 274-78.

Kanda K, Nishi K, Kadota A, Nishimoto S, Liu MC, Sugahara T. Nobiletin suppresses adipocyte differentiation of 3T3-L1 cells by an insulin and IBMX mixture induction. Biochim Biophys Acta. 2012; 1820: 461-68.

Kang JW, Nam D, Kim KH, Huh JE, Lee JD. Effect of gambisan on the inhibition of adipogenesis in 3T3-L1 adipocytes. Evid Based Complement Alternat Med. 2013a; 2013.

Kang SW, Kang SI, Shin HS, Yoon SA, Kim JH, Ko HC, Kim SJ. Sasa quelpaertensis Nakai extract and its constituent pcoumaric acid inhibit adipogenesis in 3T3-L1 cells through activation of the AMPK pathway. Food Chem Toxicol. 2013b; 59: 380-85.

Kwak DH, Lee JH, Kim DG, Kim T, Lee KJ, Ma JY. Inhibitory effects of Hwangryunhaedok-Tang in 3T3-L1 adipogenesis by regulation of Raf/MEK1/ERK1/2 pathway and PDK1/ Akt Phosphorylation. Evid Based Complement Alternat Med. 2013; 2013.

Kwak DH, Lee JH, Song KH, Ma JY. Inhibitory effects of baicalin in the early stage of 3T3-L1 preadipocytes differentiation by down-regulation of PDK1/Akt phosphorylation. Mol Cell Biochem. 2014; 385: 257-64.

Lampe MA, Burlingame AL, Whitney J, Williams ML, Brown BE, Roitman E, Elias PM. Human stratum corneum lipids: Characterization and regional variations. J Lipid Res. 1983; 24: 120-30.

Lee M, Lee SH, Kang J, Yang H, Jeong EJ, Kim HP, Kim YC, Sung SH. Salicortin-derivatives from salix pseudo-lasiogyne twigs inhibit adipogenesis in 3T3-L1 cells via modulation of C/EBPa and SREBP1c dependent pathway. Molecules 2013; 18: 10484-96.

Ogden CL, Carroll MD, Kit BK, Flegal KM. Prevalence of childhood and adult obesity in the united states, 2011-2012. JAMA. 2014; 311: 806-14.

Song Y, Park HJ, Kang SN, Jang SH, Lee SJ, Ko YG, Kim GS, Cho JH. Blueberry peel extracts inhibit adipogenesis in 3T3L1 cells and reduce high-fat diet-induced obesity. PLoS One. 2013; 8: e69925.

Spiegelman BM, Flier JS. Adipogenesis and obesity: Rounding out the big picture. Cell 1996; 87: 377-89. 
Xing Y, Yan F, Liu Y, Liu Y, Zhao Y. Matrine inhibits 3T3-L1 preadipocyte differentiation associated with suppression of ERK1/2 phosphorylation. Biochem Biophys Res Commun. 2010; 396: 691-95.

Zhang Y, Liu X, Han L, Gao X, Liu E, Wang T. Regulation of lipid and glucose homeostasis by mango tree leaf extract is mediated by AMPK and PI3K/AKT signaling pathways. Food Chem. 2013; 141: 2896-905.

Zimmet PZ, Magliano DJ, Herman WH, Shaw JE. Diabetes: A 21st century challenge. Lancet Diabetes Endocrinol. 2014; 2: $56-64$.

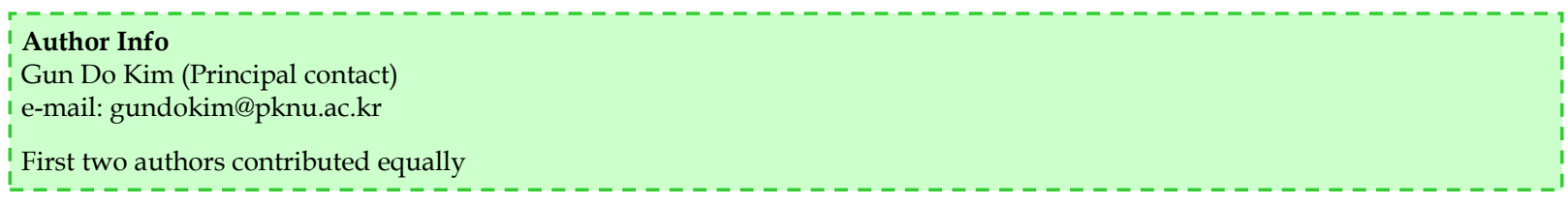

